\title{
The Efficacy of a Home-Based Nursing Program in Diabetic Control of Elderly People with Diabetes Mellitus Living Alone
}

\author{
Chun-Lien Huang, M.S.N., R.N., \\ Shiao-Chi Wu, Ph.D., \\ Chii-Yuan Jeng, M.D., Ph.D., and \\ Li-Chan Lin, R.N., Ph.D.
}

Abstract The purpose of this study was to evaluate the efficacy of a home-based nursing program in the diabetic control of elderly people with diabetes mellitus living alone. Patients meeting the sampling criteria were recruited from a medical center and 10 health centers in Taipei for this quasi-experimental study. By matching the effects of age, sex, education, and history of diabetes, subjects were assigned semirandomly to two groups based on the intensity of home-based nursing care visitations. Group I was defined as daily visits to supervise diet, exercise, medication, and self-monitoring blood sugar $(n=15)$ and Group II as weekly visits to supervise diet, exercise, medication education, and self-monitoring blood sugar $(n=15)$. Patients who agreed only to receive blood examination were assigned to the control group $(n=14)$. The results of the study showed that reductions in fasting blood sugar, postmeal blood sugar, and hemoglobin Alc (HbAlc) in Groups I and II were significantly greater than those in the control group. The reduc-

Chun-Lien Huang is Head Nurse, Nursing Department, Tri-Service General Hospital, Taipei, Taiwan. Shiao-Chi Wu is Associate Professor, Institute of Health and Social Welfare Policy, National Yang-Ming University, Taipei, Taiwan. Chii-Yuan Jeng is Associate Professor, Graduate School of Medical Science, Division of Endocrinology and Metabolism, Department of Medicine, Tri-Service General Hospital, National Defense Medical Center, Taipei, Taiwan. Li-Chan Lin is Professor, Institute of Clinical Nursing, National Yang-Ming University, Taipei, Taiwan.

Address correspondence to Li-Chan Lin, Institute of Clinical Nursing, National Yang-Ming University, 155 Li-Nong Street, Sec 2, Taipei, Taiwan.E-mail:lichan@ym.edu.tw tion in the total cholesterol and low-density lipoprotein in Groups I and II was significantly greater than that in the control group. There were no significant differences among the three groups in the improvement of high-density lipoprotein (HDL) and triglycerides (TGs). Group I revealed a significantly greater weight reduction compared to Group II. There was no significant difference between Groups I and II in the improvements of diabetes knowledge, depression level, or quality of life. From the research findings, based on cost-effectiveness, it is recommend that Program II be implemented.

Key words: blood lipid, body weight control, elderly people living alone, glycemic control, home-based nursing, quality of life.

Proper glycemic control can improve cognitive function, quality of life, psychological status, and well-being of patients with diabetes. It can lessen stress, anxiety, and depression, as well as reduce medical complications, hospitalization, mortality rates, and medical costs (The Diabetes Control and Complications Trial Research Group, 1993; Andersson \& Svardsudd, 1995; Lincoln, Kirk, Faleiro, Jeffcoate, \& Kelly, 1996; Van Der Does et al., 1996; American Diabetes Association, 1998; Testa \& Simonson, 1998; Goddijn et al., 1999). Although subjects who adhered to self-care had better metabolic control than those who neglected self-care, poor control of blood sugar may cause dangerous complications (The 
Diabetes Control and Complications Trial Research Group, 1993; Toljamo \& Hentinen, 2001). Zhan (1983) pointed out that less than $50 \%$ of diabetics in Taiwan received appropriate diet, exercise, body weight control, or even medical treatment. Wang and Liu (2001) also pointed out that only $39.33 \%$ of diabetics in Taiwan had appropriate exercise behavior. Only $20.3 \%$ of diabetics in Taiwan achieved good glycemic control among community-dwelling residents (Chong \& Lin, 2000).

The above studies reveal that no more than half of community-dwelling patients with diabetes in Taiwan comply with an appropriate diet, exercise, and medical regimen. Compliance is affected by the state of psychological health, including fear, depression, anxiety, etc. (Shillitoe \& Christie, 1990). Studies show that the state of psychological health in elderly people living alone is worse than that of those living with others (Mui \& Burnette, 1994), because they are more susceptible to factors leading to a lower quality of life, such as poverty, social separation, loneliness, and deteriorating health (Gurley, Lum, \& Katz, 1996; Yamashita, Iijima, \& Kobayashi, 1999; Mahoney, Eisner, Havighurst, Gray, \& Palta, 2000). Thus, there is rationale to assume that elderly people living alone are associated with self-care neglect.

Good glycemic control depends on triple coordination of diet, exercise, and medication. The dietary caloric distribution recommended by American Diabetes Association (1997) is $50 \%$ of calories from carbohydrate, approximately $20 \%$ from protein, and approximately $30 \%$ from fat. Several studies have demonstrated that a high-carbohydrate, low-fat diet can control blood sugar and also help lower lipid, cholesterol, and risk factors of cardiovascular complications (Garg, Bonanome, Grundy, Zhang, \& Unger, 1988; Milne, Mann, Chisholm, \& Williams, 1994). Studies have shown the effect of exercise on glycemic control. The exercises recommended include walking, cycling, jogging, and treadmill cycling. The exercise regimen is $20-60 \mathrm{~min}$ per session for programs ranging from 7 days to 15 weeks in length. The frequency of exercise is typically three to five times per week for longer programs, the 7-day intensive course having daily sessions. All studies have shown that exercise can achieve diabetes control through improving glucose tolerance and insulin sensitivity, decreasing body weight, lowering blood sugar and hemoglobin Alc (HbAlc), and improving metabolism (Hornsby et al., 1990; Braun, Zimmermann, \& Kretchmer, 1995; Taniguchi et al., 2000; Ku \& Jeng, 2001). Although diet or exercise by themselves can help control diabetes, studies have shown that a combined therapy of diet and exercise has better results. These were significant decreases of fasting blood sugar, total cholesterol, triglycerides (TGs), and body weight
(Barnard, Jung, \& Inkeles, 1994; Barnard, Ugianskis, \& Martin, 1992; Yamanouchi et al., 1995).

Self-monitoring of blood sugar can provide feedback to change patients' behavior and improve compliance (Gilden, Casia, Hendryx, \& Singh, 1990; Funnell \& Merritt, 1993). Gilden et al. (1990) studied elderly diabetics, aged between 60 and 79 years, who were taught how to selfmonitor their blood sugar. Ten subjects self-monitored blood sugar and another 10 self-monitored urine sugar. Results showed that subjects who self-monitored blood sugar were more likely to adhere to medication orders and found that this method was easier to fit into the subjects' personal schedules, easier to record, less embarrassing, and entailed no interference in quality of life. Self-monitoring of blood sugar provided reference data for medical staff and acted to encourage and interest patients who could easily see the results of their own monitoring.

Elderly people with diabetes mellitus living alone experience a unique challenge because of the physical and functional changes imposed by the aging process and the prevalence of multiple chronic illnesses and complications. They cannot independently perform self-care such as meal planning, insulin injection, self-monitoring of blood sugar, etc. (Funnell, 1990; Pritchard, 1996).

It is most important to investigate how to assist elderly people with diabetes mellitus living alone improve their glycemic control. The purpose of this study was to evaluate the efficacy of a home-based nursing program in diabetic control of elderly people with diabetes mellitus living alone.

\section{RESEARCH DESIGN AND METHODS}

\section{Procedures}

This study used a quasi-experimental design with pre- and postprogram medical examinations on two experimental groups and one control group.

In the first experimental group (Group I), participants received "home-based nursing Program I" for 6 weeks. Program I consisted of daily visits (by the research assistant who was trained by the principal investigator) to supervise subjects' diet preparation (50-60\% carbohydrate, $30 \%$ fat, and $10-20 \%$ protein), exercise performance (walking for $30 \mathrm{~min}$ three times per week and pulse rate to $50-70 \%$ maximum heart beat), medication, and self-monitoring of blood sugar (to enhance medication motivation). The second experimental group (Group II) participants received "nursing Program II," which provided health education information and self-monitoring of blood sugar, also for 6 weeks. The educational material 
was based on a booklet, "Health Education For Diabetes Mellitus Patients About Diet, Exercise, Medicine and Self-monitoring Of Blood Sugar," compiled by the investigators. The educational material was presented to Group II subjects during 20-30-min sessions held once per week. During the 6-week program, postmeal blood sugar was checked every week.

For the control group, no specific nursing program was provided, but blood sugar and lipids were checked prior to and after the 6-week program of Groups I and II. The principal investigator performed all the procedures, and a research assistant collected the data. The postmeal sugar assay of each group was arranged at the same time to lower the effect of interference. The research assistant also assisted the subjects in recording their progress notes, such as the contents of each meal, exercise performance, and daily blood sugar.

\section{Sampling}

This study used convenience sampling, and a total of 44 subjects who agreed to participate in the study were included. Thirty-five of a possible 171 subjects were selected from records from Taipei City District Health Centers, and nine of a possible 1262 subjects were selected from the metabolic department of a medical center in Taipei City. The subjects selected had to meet the following criteria: (1) be diagnosed with diabetes mellitus and living alone; (2) be Mandarin and Taiwanese speakers; (3) be 65 years or older; and (4) their blood pressure at rest less than $160 / 100 \mathrm{mmHg}$, pulse rate less than $90 / \mathrm{min}$, and respiratory rate less than $25 / \mathrm{min}$. By matching the effects of age ( \pm 2 years), sex, education, and history of diabetes, subjects were assigned semirandomly to the "home-based nursing program" (Group I, $n=15$ ) or the "education program" (Group II, $n=15$ ). Patients who agreed to only receive the blood examination were assigned to the control group $(n=14)$. All subjects were aware that they had entered into the study.

\section{Instruments}

This study used a Precision Q.I.D. machine to assay blood sugar. Concurrent validity measured for this machine was $r=0.984$. To determine the consistency of the machine's performance, 10 healthy adults had their blood sugar separately assayed with blood from their index and middle fingers. The Wilcoxon signed rank test was used to compare the two data sets. The result showed no statistical significance $(p>0.05)$. The intraclass correlation coefficient (ICC) was also used to compare the two data sets, yielding an ICC of 0.83 , indicating a modest reliability. A DCA2000TM was used to check HbA1c, a
DRI-CHEM FDC 3000 was used to measure blood lipid, and a CAMRY Health Meter measured fasting body weight. All blood samples were assayed by the same machines, which were all calibrated by a professional technician. The principal investigator performed all procedures.

The Diabetes Knowledge Scale in Chinese was devised by the investigators from available literature and health education booklets used in the "home-based nursing program." There were 12 multiple-choice questions, with a higher score indicating a higher level of diabetes knowledge. Seven experts (including metabolic specialists, nurses, nutritionists, and associate professors) were invited to determine the content validity of the Diabetes Knowledge Scale. Validity was measured using the Likert four-point method, with scores ranging from very inappropriate (one point) to very appropriate (four points). The average score was above three points, and the content validity index (CVI) was 1.0. Forty-eight hospitalized diabetic patients from the metabolic department at a different medical center were used to determine internal consistency; the KR 20 was 0.793 , indicating modest internal consistency.

A Chinese language version of the Depression Scale was developed by Hsu (1993) and consists of 20 questions evaluated by the Likert four-point method. Cronbach's $\alpha$ of the scale was 0.878 . A higher score indicates the greater feeling of depression the subject has. The CVI from the seven experts was $100 \%$, indicating a good content validity. Cronbach's $\alpha$ from 48 hospitalized diabetic patients was 0.878 , indicating modest internal consistency.

A Quality of Life Scale was revised from Liu's (1993) "Quality of Life Index," Chinese language edition. The criterion validity in the original scale was $r=0.77$, and the subscales' Cronbach's $\alpha$ ranged from 0.80 to 0.95 . There were 15 questions after revision that were evaluated by the Likert four-point method and reflected aspects of importance and satisfaction. Each question was used to score the feelings of importance in life, which ranged from unimportant (one point) to very important (four points). Each question was also scored for satisfaction in life, with scores ranging from very unsatisfactory $(-1.5$ points) to very satisfactory $(+1.5$ points). The total score for each question was the satisfaction score $(-1.5,-0.5,+0.5$, or $+1.5)$ multiplied by the importance score $(1,2,3$, or 4$)$. The scores for each question answered were then averaged. To prevent negative score values, six points were added to the score (lowest possible score for any question was -6 , highest +6 ). This resulted in a possible average value ranging from 0 to 12 , with higher values indicating a better quality of life. Cronbach's $\alpha$ for the Quality of Life Scale from 48 hospitalized diabetics was 0.902. 


\section{Statistical Analysis}

The data were analyzed using descriptive analysis (frequency, percentage, mean, and standard deviation) and Wilcoxon signed rank, Mann-Whitney $U$ test, KruskalWallis, and chi-square tests.

\section{RESULTS}

The subject's average age in this study was 76.2 years, ranging from 66 to $89(S D=6.0)$. Twenty-four subjects were male $(54.5 \%) ; 70.4 \%$ had been diabetics for more than 10 years. Forty-two of the subjects $(95.5 \%)$ diagnosed with type 2 diabetes were being treated by oral hypoglycemic agents (Table 1). Demographic data of the three study groups were analyzed using the KruskalWallis test, which showed no significant differences, implying that the three groups were homogenous (Table 1).

Subjects in Group I showed significant pre- and postprogram differences between the average values of fasting blood sugar, postmeal blood sugar, and $\mathrm{HbAlc}$ in the assays $(z=-3.408, z=-3.408, z=-3.412$, respectively, $p<0.01)$. HbAlc did not show a significant difference before and after program for Group II. Significant differences between average values of fasting blood sugar and postmeal blood sugar in the pre- and postprogram assays in Group II were also noted $(z=-2.727, z=-2.615$, respectively, $p<0.01)$. No significant difference between average values of fasting blood sugar and HbAlc in preand postprogram assays in the control group was noted $(z=0.912, z=0.528$, respectively, $p<0.05)$. The KruskalWallis test results on improvement of fasting blood sugar yielded significant results for mean differences of blood sugar and HbAlc among the three groups $(p<0.001)$. In further analysis, the Mann-Whitney $U$ test showed that the mean differences of fasting blood sugar of Groups I and II were much higher than the control group $(z=-4.214, z=-2.861, p<0.01)$. The mean difference of fasting blood sugar of Group I was higher than that of Group II $(z=-4.109, p<0.001)$. For HbAlc, the mean difference of Group I was higher than Group II $(z=-2.455, p<0.05)$, and the mean difference of Group II was much higher than the control group $(z=-2.282, p<0.05)$ (Table 2).

For blood lipid, the average values of pre- and postprogram assays of total cholesterol, high-density lipoprotein (HDL), low-density lipoprotein (LDL) and TGs in all groups showed no statistically significant differences $(p>0.05)$. The Kruskal-Wallis test was used to analyze the mean differences. The mean differences of cholesterol and LDL were statistically significant $(p<0.05)$. The Mann-Whitney $U$ test showed that the mean differences of cholesterol of Groups I and II were higher than those of control group $(z=-2.032, z=-2.599, p<0.05)$, and for improvement of LDL, the mean differences of Groups I and II were higher than those of the control group $(z=-2.536, z=-2.337, p<0.05)$ (Table 2).

The average loss of body weight before and after program was $1.9 \mathrm{~kg}$ in Group I but only $0.53 \mathrm{~kg}$ in Group II. The mean differences between the two groups were statistically significant $(z=-3.489, p=0.000)$. The average increased score of pre- to postprogram diabetes knowledge was 4.07 points in Group I compared to 3.40 points in Group II. Overall average pre- and postprogram scores of both groups were statistically significant; however, the mean difference between the two groups was not statistically significant $(z=-1.375, p=0.178)$. The level of

TABLE 1. Description of Subjects $(n=44)$

\begin{tabular}{|c|c|c|c|c|c|}
\hline & Group I & Group II & Control group & Kruskal-Wallis test & $\chi^{2}$ \\
\hline \multicolumn{6}{|l|}{$\operatorname{Sex}[n(\%)]$} \\
\hline Female & $7(46.7)$ & $7(46.7)$ & $6(42.9)$ & & \\
\hline Education $[M(S D)]$ & $5.9(5.4)$ & $5.9(5.5)$ & $6.2(3.2)$ & 0.259 & \\
\hline \multicolumn{6}{|c|}{ History of diabetes $[n(\%)]$} \\
\hline \multicolumn{6}{|l|}{ Treatment $[n(\%)]$} \\
\hline OHA & $14(93.3)$ & $14(93.3)$ & $14(100)$ & & 0.978 \\
\hline Insulin & $1(6.7)$ & $1(6.7)$ & & & \\
\hline
\end{tabular}

Note. OHA, oral hypoglycemic agents. 
TABLE 2. Comparison of Mean Differences in Pre- and Post-Test of Blood Sugar and Lipid Among the Three Groups ( $n=44$ )

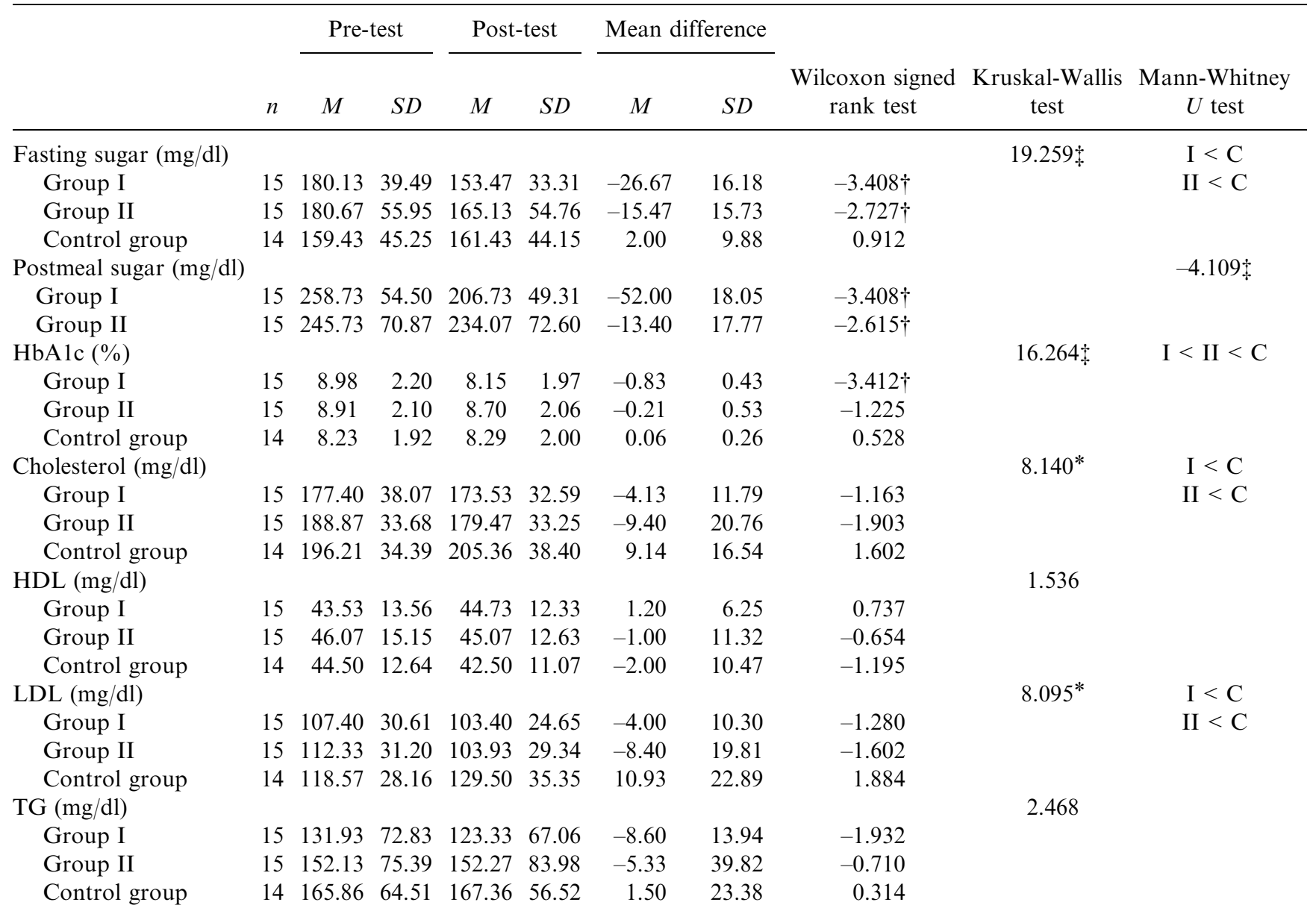

Note. HbA1c, hemoglobin A1c; HDL, high-density lipoprotein; LDL, low-density lipoprotein; TG, triglyceride. I, Group I; II, Group II; C, control group.

$*_{p}<0.05$.

$\dagger p<0.01$.

$\dagger p<0.001$.

depression showed an average score decrease before and after program of 1.07 points in Group I and 4.07 points in Group II. The mean difference of pre- and postprogram scores of both groups was not statistically significant $(z=-1.933, p=0.056)$. The quality of life average score increase before and after program was 0.64 points in Group I and 0.37 points in Group II. The mean difference of Group I was only 0.27 points higher than that of Group II and showed no statistical significance $(z=-1.017, p=0.325)$. In Group I, which received daily management, the increase of the quality of life scores before and after program was statistically significant; however, Group II showed no significant difference (Table 3).

\section{DISCUSSION}

This study showed that the mean differences of fasting blood sugar, postmeal blood sugar, and $\mathrm{HbAlc}$ of the three groups were statistically significant. Results are consistent with those from studies of Barnard et al. (1994), who used a larger sample size $(n=652)$, and of Yamanouchi et al. (1995), who used a smaller sample size $(n=24)$. Although the mean differences of Groups I and II were not statistically significant for fasting blood sugar, the decrease in Group I was greater than Group II and approached significance $(p=0.089)$. Mean differences of postmeal blood sugar and HbAlc were statistically significant between Groups I and II. Avignon, Radauceanu, and Monnier (1997) reported that postmeal sugar is better 
TABLE 3. Comparison of Mean Differences in Pre- and Post-Test of Body Weight, Diabetes Knowledge, Depression Level, and Quality of Life Among the Three Groups $(n=30)$

\begin{tabular}{|c|c|c|c|c|c|c|c|c|c|}
\hline & $n$ & \multicolumn{2}{|c|}{ Pre-test } & \multicolumn{2}{|c|}{ Post-test } & \multicolumn{2}{|c|}{ Mean difference } & Wilcoxon signed rank test $(z)$ & Mann-Whitney $U$ test $(z)$ \\
\hline Group I & 15 & 62.57 & 9.85 & 60.67 & 9.22 & -1.90 & 1.07 & $-3.426 \dagger$ & \multirow[t]{2}{*}{$-3.489 t$} \\
\hline Group II & 15 & 57.87 & 8.75 & 57.33 & 9.05 & -0.54 & 0.77 & $-2.268^{*}$ & \\
\hline \multicolumn{10}{|c|}{ Diabetes knowledge } \\
\hline \multicolumn{10}{|c|}{ Depression level } \\
\hline Group I & 15 & 12.87 & 9.30 & 11.80 & 8.33 & -1.07 & 3.41 & -1.520 & \multirow[t]{2}{*}{-1.933} \\
\hline Group II & 15 & 13.20 & 6.55 & 9.13 & 4.00 & -4.07 & 3.90 & $-3.025 \dagger$ & \\
\hline \multicolumn{10}{|c|}{ Quality of life } \\
\hline Group I & 15 & 7.67 & 2.58 & 8.31 & 2.07 & 0.64 & 0.84 & $2.385^{*}$ & -1.017 \\
\hline
\end{tabular}

$* p<0.05$.

$\dagger p<0.01$.

$\uparrow p<0.001$.

than fasting blood sugar in detecting glycemic control status. In comparison to the control group, Group II was better at improving glycemic control, implying that systematic health education, weekly visits, and monitoring of blood sugar can improve diabetics' glycemic control. If health care professionals can provide a specific diet program and supervision, the effect will be better than health education alone. If there were inadequate manpower or funding, a Program II style of protocol is recommended.

The mean differences of cholesterol and LDL between Groups I and II were much better than the control group; however, the mean differences of HDL and TGs were not statistically significant. Improvement of blood lipid in this study was not as good as that reported by Milne et al. (1994). One possible reason may be the shorter 6-week time span between pre- and postprogram assays in this study compared to the 3- to 18-month time span of Milne et al. (1994). Also, from a physiological aspect, insulin controls not only carbohydrate, but also lipid metabolism; in a short time span, the improvement of blood sugar can be identified only at an insulin-induced level, which may mask any possible improvement in TG. The metabolite of a high-carbohydrate diet will be transformed to TG, thus elevating measured levels, and a high TG will lower HDL production (Vander, Sherman, \& Luciano, 1997).

The mean differences of HDL and TG between the groups were not statistically significant. The average pre- and postprogram HDL values increased $(1.20 \mathrm{mg} /$ dl) in Group I but decreased in Group II and the control group. The mean differences of TG decreased much more in Group I than in Group II and increased in the control group. This indicates that "home-based nursing Program I" improved HDL and TG in some manner still to be identified.

For body weight, the mean difference of Group I was much higher than that of Group II $(p<0.001)$, indicating that Program I is better than Program II. This result is consistent with the work of Braun et al. (1995) that showed that exercise was effective in helping reduce body weight.

The mean differences of diabetes knowledge between Groups I and II were not statistically significant. Within both Groups I and II, the difference between the pre- and postprogram assays scores, however, was statistically significant, indicating that both "home-based nursing Program I" and "nursing education Program II" improved diabetes knowledge in elderly diabetics living alone.

For level of depression, the mean differences between Groups I and II were not statistically significant; however, the decrease was less in Group II than Group I. This might be due to learning problems associated with using the blood sugar monitoring machine and diet change. For example, one subject complained "It is useless once you get old. I cannot do this triviality (self-monitoring blood sugar)." This affected the subjects' level of depression. Although improvement of the level of depression between the two groups was not statistically significant, the postprogram assays were lower than those of the preprogram assay, and glycemic control improved within both groups. 
This supports the results of Mazze, Lucido, and Shamoon (1984), who showed that better glycemic control lowered depression levels.

The mean differences for the quality of life scores between Groups I and II were not statistically significant, which might be due to the small sample size. The reason why this study was unable to recruit large sample size is that only $12.28 \%$ of elderly people live alone in Taiwan (Ministry of the Interior, 1997), due to the cultural tradition of elderly people living with family members. Thus, in spite of the fact that 1,433 elderly people with diabetes were screened, only 44 subjects met the sampling criteria and agreed to participate in this study. Even though the mean differences of the quality of life scores between Groups I and II were not statistically significant, the postprogram assay score was higher than the preprogram score within Group I and was statistically significant. Within Group II, the pre- and postassay scores did not have a statistically significant difference. This implies that "home-based nursing Program I" did improve quality of life for elderly diabetics living alone. In addition, the improvement of glycemic control was much better in Group I than in Group II. These findings are supported by the studies of Testa and Simonson (1998) and Goddijn et al. (1999) which showed that better glycemic control resulted in an improvement of quality of life. Although the mean differences of quality of life in this study were not statistically significant, the within-groups differences imply that the better the improvement of blood sugar, the better the improvement in quality of life.

\section{CONCLUSIONS}

Because of time and sample size limitations, some variables were not significant; however, most biochemical data such as fasting sugar, postmeal sugar, HbA1c, cholesterol, and LDL were significant. Results demonstrate that a "home-based nursing program" can improve glycemic control of elderly diabetics living alone. This can be extended to the public health nursing arena as a means to improve current care programs for elderly diabetics living alone. If there is ample personnel available for daily at-home supervision, Program I is optimal. Because of the demand for staff, however, Program I is difficult to implement immediately in the public health field. Fortunately, even though the effects of Program II were not ideal, it still showed much better improvement than the control group. It is recommended that Program II, consisting of weekly visits and blood sugar monitoring assistance for elderly diabetics living alone, be initially implemented into the public health care system. Program II could provide an improvement in systemic health education that is more cost effective and has a good likelihood of current popular acceptance. With the establishment of an adequate manpower resource, Program I can be implemented to help elderly diabetics living alone to improve their glycemic control to an even greater level.

\section{ACKNOWLEDGMENTS}

The authors thank the metabolic department of the TriService General Hospital for support of laboratory equipment and all the elderly diabetics who participated in the study. Without their help, it would have been impossible to complete this study.

\section{REFERENCES}

American Diabetes Association. (1997). Translation of the diabetes nutrition recommendations for health care institutions. Diabetes Care, 20(1), 106-108.

American Diabetes Association. (1998). Economic consequences of diabetes mellitus in the U.S. in 1977. Diabetes Care, 21(2), 296-309.

Andersson, D. K. G. \& Svardsudd, K. (1995). Long-term glycemic control relates to mortality in type a diabetes. Diabetes Care, 18(12), 1534-1543.

Avignon, A., Radauceanu, A., \& Monnier, L. (1997). Nonfasting plasma glucose is a better marker of diabetic control than fasting plasma glucose in type 2 diabetes. Diabetes Care, 20(12), 1822-1826.

Barnard, R. J., Jung, T., \& Inkeles, S. B. (1994). Diet and exercise in the treatment of NIDDM. Diabetes Care, 17, 1469-1472.

Barnard, R. J., Ugianskis, E. J., \& Martin, D. A. (1992). The effects of an intensive diet and exercise program on patients with non-insulin-dependent diabetes mellitus and hypertension. Journal of Cardiopulmonary Rehabilitation, 12, 194-201.

Braun, B., Zimmermann, M. B., \& Kretchmer, N. (1995). Effects of exercise intensity on insulin sensitivity in women with non-insulin-dependent diabetes mellitus. Journal of Applied Physiology, 78(1), 300-306.

Chong, Y. F. \& Lin, H. D. (2000). The effects of health education on knowledge, attitude and practice in seventy-four type 2 diabetic patients. Endocrinology and Diabetology, 13, 1-17.

Funnell, M. M. (1990). Role of the diabetes educator for older adult. Diabetes Care, 13(Suppl. 2), 60-65.

Funnell, M. M. \& Merritt, J. H. (1993). The challenges of diabetes and older adults. Nursing Clinics of North America, 28(1), 45-60.

Garg, A., Bonanome, A., Grundy, S. M., Zhang, Z. J., \& Unger, R. H. (1988). Comparison of a high-carbohydrate diet with a high-monounsaturated-fat diet in patients with non-insulindependent diabetes mellitus. The New England Journal of Medicine, 319(13), 829-834.

Gilden, J. L., Casia, C., Hendryx, M., \& Singh, S. P. (1990). Effects of self-monitoring of blood glucose on quality of life 
in elderly diabetes patients. Journal of the American Geriatrics Society, 38, 511-515.

Goddijn, P. P., Bilo, H. J., Feskens, E. J., Groeniert, K. H., Van Der Zee, K. I., \& Meyboom-de, J. B. (1999). Longitudinal study on glycemic control and quality of life in patients with type 2 diabetes mellitus referred for intensified control. Diabetic Medicine, 16(1), 23-30.

Gurley, R. J., Lum, N., \& Katz, M. H. (1996). Persons found in their homes helpless or dead. The New England Journal of Medicine, 334(26), 1710-1716.

Hornsby, W. G., Boggess, K. A., Lyons, T. J., Barnwell, W. H., Lazarhick, J., \& Colwell, J. A. (1990). Hemostatic alterations with exercise conditioning in NIDDM. Diabetes Care, 13(2), 87-92.

Hsu, Y. Z. (1993). Correlation study of the attitudes toward old age, social support, self-reported health condition and depression of the age between 65 and 74 years old adults at Lukang community. Graduate Institute of Health Education Master Thesis, National Taiwan Normal University.

$\mathrm{Ku}, \mathrm{C}$. T. \& Jeng, C. (2001). Impact of different activity modes and durations on immediate serum glucose response among type 2 diabetes mellitus patients. Nursing Research (Chinese), 9(3), 203-212.

Lincoln, N. B., Kirk, B. A., Faleiro, R. M., Jeffcoate, W. J., Kelly, C. (1996). Effect of long-term glycemic control on cognitive function. Diabetes Care, 19(6), 656-658.

Liu, H. E. (1993). An exploration of the psychometrics of the quality of life index of Chinese family. Nursing Research (Chinese), 1(2), 127-136.

Mahoney, J. E., Eisner, J., Havighurst, T., Gray, S., \& Palta, M. (2000). Problems of older adults living alone after hospitalization. Journal of General Internal Medicine, 15(9), 611-619.

Mazze, R. S., Lucido, D., \& Shamoon, H. (1984). Psychological and social correlates of glycemic control. Diabetes Care, 7(4), 360-366.

Milne, R. B., Mann, J. I., Chisholm, A. W., \& Williams, S. M. (1994). Long-term comparison of three dietary prescriptions in the treatment of NIDDM. Diabetes Care, 17(1), 74-80.

Ministry of the Interior. (1997). The conditions of elderly people, 1996. Taipei, Taiwan: Department of Statistics, Ministry of the Interior.

Mui, A. C., \& Burnette, J. D. (1994). A comparative profile of frail elderly persons living alone and those living with others. Journal of Gerontological Social Work, 21(3/4), 5-26.
Pritchard, C. E. (1996). Update in diabetes: Applications for clinical practice from the diabetes control and complications trial. Nursing Clinics of North America, 31(4), 725-735.

Shillitoe, R. \& Christie, M. (1990). Psychological approaches to the management of chronic illness: the example of diabetes mellitus. In P. Bennett, J. Weinman, \& P. Spurgeon (Eds.), Current developments in health psychology (pp. 177-208). London: Harwood Academic.

Taniguchi, A., Fukushima, M., Sakai, M., Nagasaka, S., Doi, K., Nagata, I., et al. (2000). Effect of physical training on insulin sensitivity in Japanese type 2 diabetic patients: role of serum triglyceride levels. Diabetes Care, 23(6), 857-858.

Testa, M. A. \& Simonson, D. C. (1998). Health economic benefits and quality of life during improved glycemic control in patients with type 2 diabetes mellitus: a randomized, controlled, double-blind trial. JAMA, 280(17), 1490-1496.

The Diabetes Control and Complications Trial Research Group. (1993). The effect of intensive treatment of diabetes on the development and progression of long-term complications in insulin-dependent diabetes mellitus. The New England Journal of Medicine, 329(14), 977-986.

Toljamo, M., \& Hentinen, M. (2001). Adherence to self-care and glycaemic control among people with insulin-dependent diabetes mellitus. Journal of Advanced Nursing, 34(6), 780786.

Van Der Does, F. E. E., Grootenhuis, P. A., Neeling, J. N. D., Bouter, L. A., Snoek, F. J., Heine, R. J. et al. (1996). Symptoms and well-being in relation to glycemic control in type a diabetes. Diabetes Care, 19(3), 204-210.

Vander, A. J., Sherman, J. H., \& Luciano, D. S. (1997). Human physiology. New York: McGraw-Hill.

Wang, M. S. \& Liu, S. J. (2001). Effects of the supportive exercise teaching program on diabetes. Veterans General Hospital-Taipei Nursing, 18(3), 259-269.

Yamanouchi, K., Shinozaki, T., Chikada, K., Nishikawa, T., Ito, K., Shimizu, S., et al. (1995). Daily walking combined with diet therapy is a useful means for obese NIDDM patients not only to reduce body weight but also to improve insulin sensitivity. Diabetes Care, 18(6), 775-778.

Yamashita, K., Iijima, K., \& Kobayashi, S. (1999). Relationship among activities of daily living, apathy, and subjective wellbeing in elderly people living alone in a rural town. Gerontology, 45(5), 279-282.

Zhan, H. M. (1983). The treatment and related factors of patients with diabetes mellitus at home in one hospital, Taipei. Public Health, 10(3), 256-272. 